\title{
Sorafenib for the treatment of hepatocellular carcinoma with portal vein tumour thrombosis: a systematic review of comparative studies
}

\author{
Xingshun Qi, Xiaozhong Guo \\ Department of Gastroenterology, General Hospital of Shenyang Military Area, Shenyang, China
}

Prz Gastroenterol 2015; 10 (3): 142-147 DOI: $10.5114 /$ pg.2015.52470

Key words: sorafenib, hepatocellular carcinoma, portal vein tumour thrombosis, radiofrequency ablation, cryotherapy, transarterial chemoembolization, radioembolisation, radiotherapy.

Address for correspondence: Xingshun Qi MD, Department of Gastroenterology, General Hospital of Shenyang Military Area, No. 83 Wenhua Road, 110840 Shenyang, China, phone: +86 29 84771537, e-mail: xingshunqi@126.com; Xiaozhong Guo MD, PhD, e-mail: guo_xiao_zhong@126.com

\begin{abstract}
Sorafenib is the first-line treatment of choice for advanced hepatocellular carcinoma (HCC). However, the benefits of sorafenib in HCC patients with portal vein tumour thrombosis (PVTT) remain uncertain. Until now, a total of eight comparative studies have been identified for this systematic review. Four retrospective studies showed that hepatic arterial infusion chemotherapy, hepatic resection, and three-dimensional conformal radiotherapy might be superior to sorafenib in improving the overall survival. Two ongoing randomised controlled trials (RCTs) will compare the outcomes of transarterial chemoembolisation or radioembolisation with those of sorafenib for the treatment of HCC with PVTT. In addition, two completed RCTs found that additional use of cryotherapy or radiofrequency ablation could prolong the survival of patients receiving sorafenib. In conclusion, the clinical efficacy of sorafenib in HCC patients with PVTT has been widely challenged by other interventions. However, further well-designed RCTs are necessary to confirm the findings of retrospective analyses. Cryotherapy or radiofrequency ablation may be considered as an adjunctive therapy in such patients, if sorafenib is prescribed.
\end{abstract}

\section{Introduction}

Hepatocellular carcinoma (HCC) is the third most common cause of cancer-related death [1]. The presence of portal vein tumour thrombosis (PVTT) is regarded as one of the most important prognostic factors in HCC patients [2]. However, there is no consensus about the treatment of HCC with PVTT. According to the Barcelona Clinic Liver Cancer (BCLC) staging system [3], HCC patients with PVTT should be at the advanced stage, and in whom sorafenib should be recommended as the first-line treatment modality. This recommendation is primarily based on the results of two randomised, double-blinded, controlled trials, which showed that the use of sorafenib can achieve a significant survival benefit in patients with advanced HCC $[4,5]$. Notably, in the two randomised controlled trials (RCTs), only $30-40 \%$ of patients had macroscopic vascular invasion, and the site of vascular invasion was unclear. In the SHARP trial by Llovet et al., $36 \%$ and $41 \%$ of patients receiving sorafenib and placebo had macroscopic vascular inva- sion, respectively. In the ORIENTAL trial by Cheng et al., $36 \%$ and $34.2 \%$ of patients receiving sorafenib and placebo had macroscopic vascular invasion, respectively. In addition, scatter case reports suggested that complete portal vein recanalisation could develop after sorafenib in HCC patients with PVTT [6-9]. However, the benefits of sorafenib in HCC patients with PVTT have been questioned because the presence of PVTT significantly decreases the survival of HCC patients receiving sorafenib [10-13]. On the other hand, until now, the role of other alternative treatment modalities versus sorafenib in such patients remains unclear. Considering that the knowledge is needed to guide the clinical decisions, we have conducted a systematic review of available studies comparing the efficacy of sorafenib versus other interventions in HCC patients with PVTT.

\section{Literature search and identification \\ The PubMed, EMBASE, and Cochrane library databas- es were searched for the retrieval of all relevant papers.}


In addition, the official website www.ClinicalTrials.gov was searched for the retrieval of all ongoing trials. The search items used were: "sorafenib" and "hepatocellular carcinoma" and "portal vein thrombosis". No limitations were specified for the publication language or status. Comparative studies were included if they performed a head-to-head comparison between sorafenib monotherapy and other interventions or a comparison between sorafenib alone versus combined with other interventions for the treatment of HCC with PVTT. Considering the heterogeneity of the study population and treatment modalities, we did not perform any meta-analyses.

A total of 272 papers and six trials were identified. Finally, eight comparative studies were eligible for systematic review (Figure 1) [14-21]. Characteristics and eligibility criteria of these included studies are described in Tables I and II, respectively.

\section{Sorafenib monotherapy versus other interventions}

\section{Sorafenib versus hepatic arterial infusion chemotherapy (HAIC)}

Two retrospective studies compared the outcomes of sorafenib versus HAIC in HCC patients with PVTT [17, 18]. The first one was a single-centre study conducted in Japan with 40 cases [17]. In the HAIC group, 5-fluorouracil was employed as a chemotherapeutic agent, and pegylated interferon $\alpha 2 b$ was also subcutaneously administered. The investigators found that the objective early response rate was higher in the HAIC group than in the sorafenib group $(71.4 \%$ vs. $10.5 \%, p<0.01)$. More importantly, the survival rate was higher in the HAIC group than in the sorafenib group (6-month survival rate: $83.8 \%$ vs. $68.4 \%$; 12 -month survival rate: $77.8 \%$ vs. $37.7 \%$; 18 -month survival rate: $55.6 \%$ vs. $16.2 \%$, $p=0.03)$. However, because the study was published in the abstract form, we could not evaluate the comparability of baseline characteristics between the two groups.

The second one was a multi-centre study conducted in South Korea with 110 cases [18]. In the HAIC group, the chemotherapeutic agents included cisplatin and 5-fluorouracil with or without epirubicin. The baseline Child-Pugh class and location of PVTT were comparable between the two groups, but the HAIC group enrolled a higher proportion of patients who underwent the combined loco-regional treatment than the sorafenib group. Compared with the sorafenib group, the HAIC group achieved more significant clinical efficacies, including a higher treatment response rate (complete response + partial response: $24 \%$ vs. $13.3 \%, p=0.214$ ), a higher disease control rate ( $90 \%$ vs. $45 \%, p<0.001)$, a longer overall survival time (median: 7.1 months vs. 5.5 months, $p=0.011$ ), and a longer time to progression (median: 3.3 months vs. 2.1 months, $p=0.034$ ). However, the multivariate analysis did not identify HAIC as a significant prognostic factor. Therefore, well-designed RCTs are warranted to compare the advantages of HAIC with those of sorafenib in such patients.

\section{Sorafenib versus transarterial chemoembolization (TACE)}

High-level evidence from randomised controlled trials and meta-analysis has shown that TACE can significantly improve the survival of HCC patients [22]. According to the BCLC staging system, TACE is recommended as the first-line treatment option for the intermediate stage of HCC $[3,23]$. Traditionally, the presence of PVTT is a relative contraindication for TACE, because the simultaneous blockage of hepatic artery and portal vein may lead to the liver failure. However, recent studies have confirmed the safety and feasibility of TACE in such patients [24]. At present, a phase II RCT conducted in South Korea is exploring the efficacy of sorafenib and TACE in advanced HCC patients with major branch of portal vein invasion (www.ClinicalTrials.gov Identifier: NCT01480817) [21]. But this trial has two potential limitations, as follows: 1 ) the sample size needed is only

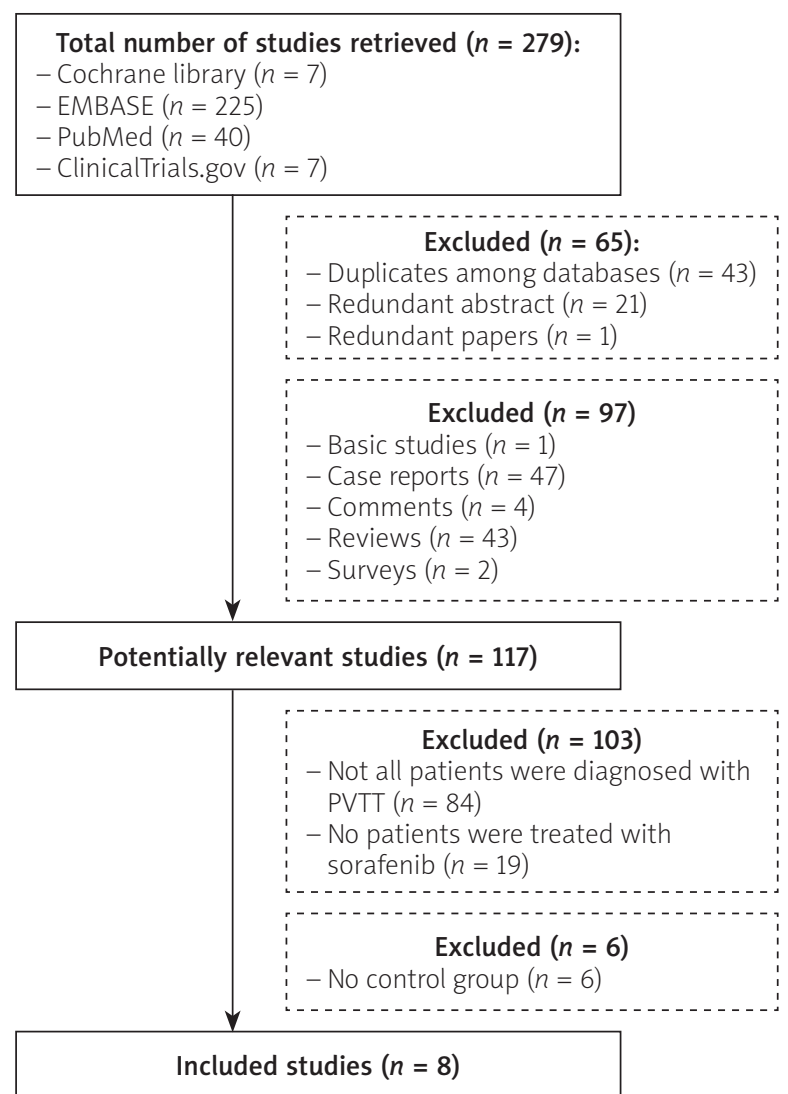

Figure 1. Flowchart of study inclusion 


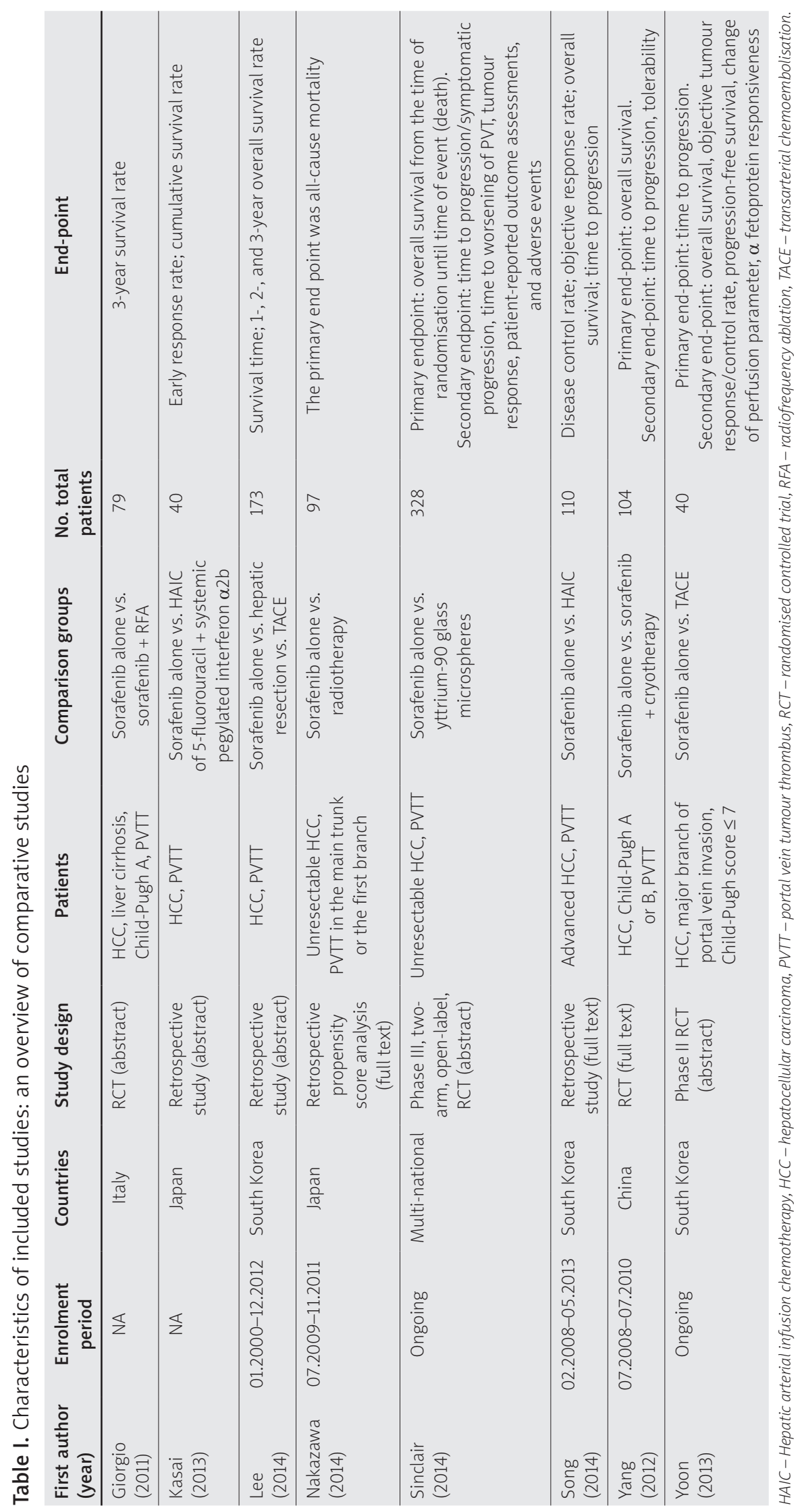


Table II. Eligibility criteria: an overview of published comparative studies

\begin{tabular}{|c|c|}
\hline $\begin{array}{l}\text { First author } \\
\text { (year) }\end{array}$ & Eligibility criteria \\
\hline Giorgio (2011) & Inclusion criteria: single HCC $(\leq 6.5 \mathrm{~cm})$ and PVTT; or $3 \mathrm{HCC}$ nodules $\leq 5 \mathrm{~cm}$ with PVTT \\
\hline Kasai (2013) & Inclusion criteria: advanced HCC patients with PVTT \\
\hline \multirow[t]{2}{*}{ Lee (2014) } & Inclusion criteria: advanced HCC patients with PVTT \\
\hline & Exclusion criteria: main portal vein tumour thrombosis, superior mesenteric vein tumour thrombosis, or Child-Pugh C \\
\hline \multirow[t]{2}{*}{ Nakazawa (2014) } & $\begin{array}{l}\text { Inclusion criteria for sorafenib: (1) unresectable advanced HCC without HCC rupture; (2) no effect of TACE; (3) no } \\
\text { previous sorafenib therapy for the liver tumour; (4) Child-Pugh A or B }(\leq 7) \text {; (5) ECOG performance status of O-2; and } \\
\text { (6) neutrophil count }>1500 / \mu \mathrm{l} \text {, PLT }>7.5 \times 10^{4} \mathrm{~mm}^{3} \text {, and Hb }>8.5 \mathrm{~g} / \mathrm{dl}\end{array}$ \\
\hline & $\begin{array}{l}\text { Inclusion criteria for radiotherapy: (1) unresectable HCC with macroscopic hepatic vascular invasion; (2) Child-Pugh } \\
\text { A or B; (3) ECOG performance status of 0-2; (4) no refractory ascites; and (5) no previous radiation therapy of the liver }\end{array}$ \\
\hline Sinclair (2014) & Inclusion criteria: PVT associated with unresectable HCC, who are not eligible for any curative procedure \\
\hline \multirow[t]{2}{*}{ Song (2014) } & $\begin{array}{l}\text { Eligibility criteria: (1) age } 18-75 \text { years; (2) radiologically confirmed PVTT in the main, first, or second branch of the } \\
\text { portal vein; (3) ECOG performance status of } 0 \text { or } 1 \text {; (4) Child-Pugh score B7; (5) WBC } \geq 3 \times 10^{9} / 1 \text { or absolute neutrophil } \\
\text { count } \geq 1.0 \times 10^{9} / \text {; and (6) PLT } \geq 50 \times 10^{9} / 1\end{array}$ \\
\hline & $\begin{array}{l}\text { Exclusion criteria: another primary tumour and other serious medical conditions, such as renal or cardiopulmonary } \\
\text { insufficiency. Patients who were treated with sorafenib in the HAIC group and those who were treated with HAIC in } \\
\text { the sorafenib group were also excluded }\end{array}$ \\
\hline Yang (2012) & $\begin{array}{l}\text { Inclusion criteria: (1) advanced HCC without distant metastasis; (2) presence of PVT; (3) ECOG performance } \\
\text { status of O, 1, or 2; (4) Child-Pugh A or B; (5) life expectancy of at least } 12 \text { weeks; (6) total bilirubin concentration of } \\
\leq 51.3 \mu \mathrm{mol} / / \text {; (7) HBV DNA positivity }\end{array}$ \\
\hline Yoon (2013) & $\begin{array}{l}\text { Inclusion criteria: (1) } 18-80 \text { years; (2) Child-Pugh class A or B }(\leq 7) \text {; (3) HCC with major branch of portal vein } \\
\text { invasion; (4) WBC } \geq 2,000 / \mu \mathrm{l} \text {, absolute neutrophil count }>1,200 / \mu \mathrm{l}, \mathrm{Hb} \geq 8.0 \mathrm{~g} / \mathrm{dl}, \mathrm{PLT}>50,000 / \mu \mathrm{l}, \mathrm{Cr}<1.7 \mathrm{mg} / \mathrm{dl} \text {, } \\
\text { TBIL } \leq 3.0 \mathrm{mg} / \mathrm{dl} \text {, PT-INR } \leq 2.3 \text { or PT } \leq 6 \mathrm{~s} \text {; (5) ECOG performance status of } 0-2\end{array}$ \\
\hline
\end{tabular}

Exclusion criteria: (1) Child-Pugh score $\geq 8$; (2) age < 18 or $\geq 80$ years; (3) ECOG performance status $\geq 3$; (4) recipient of living donor or deceased donor liver transplantation

ECOG - Eastern Cooperative Oncology Group, HAIC - hepatic arterial infusion chemotherapy, Hb-haemoglobin, HCC - hepatocellular carcinoma, PLT - platelets count, PVTT - portal vein tumour thrombus, TACE - transarterial chemoembolisation, TBIL - total bilirubin, WBC - white blood cell.

40; and 2) the primary endpoint is the time to progression, but not the overall survival.

\section{Sorafenib alone versus radioembolisation}

Transarterial radioembolisation with Yttrium-90 glass microspheres (TheraSphere ${ }^{\circledR}$ registry marker) is a promising treatment option for advanced HCC with PVTT [25-27]. A head-to-head multi-centre RCT is ongoing to compare the outcomes of sorafenib with those of radioembolisation in unresectable HCC patients with PVTT and Child-Pugh class A (www.ClinicalTrials.gov Identifier: NCT01887717) [20, 28]. This study, which is sponsored by BTG International Inc., will recruit 328 cases in four Western countries (the USA, Italy, Spain, and the UK) during a period of about 4 years. In addition, an earlier French multi-centre RCT is comparing the outcomes between the HCC patients receiving sorafenib and radioembolisation (www.ClinicalTrials.gov Identifier: NCT01482442) [29]. However, it should be noted that the HCC patients with and without PVTT will be considered as the target population.

\section{Sorafenib alone versus radiotherapy}

A Japanese retrospective study compared the outcomes of sorafenib with those of three-dimensional conformal radiotherapy in $97 \mathrm{HCC}$ patients with PVTT [14]. In the entire cohort analysis, the overall survival time was comparable between patients receiving sorafenib and radiotherapy (median: 4.4 months vs. 5.9 months, $p=0.115$ ). But the risk of bias was obvious. First, the enrolment periods were different. The patients in the sorafenib group were enrolled after 2009; by comparison, those in the radiotherapy group were enrolled after 2001. Considering that the management of advanced HCC has gradually improved over time, the selection of treatment modalities in the sorafenib group might be more appropriate. Second, the baseline characteristics were different. The sorafenib group had a significantly lower proportion of Child-Pugh class B $(10 \%$ vs. $31 \%$ ) and tumour thrombus within the main portal vein trunk ( $18 \%$ vs. $43 \%$ ) than the radiation group. To minimise the selection bias, a propensity analysis was further performed in 56 HCC patients with PVTT 
and Child-Pugh class A. The overall survival time was significantly shorter in the sorafenib group than in the radiation therapy group (median: 4.8 months vs. 10.9 months, $p=0.002$ ). This finding was also supported by the results of multivariate Cox regression analyses, which showed that radiation therapy should be identified as the independent predictor of survival (hazard ratio $=0.43,95 \%$ confidence interval: $0.235-0.779$, $p=0.007)$. These impressive findings indicated the superiority of radiation therapy over sorafenib in HCC patients with PVTT.

\section{Sorafenib versus hepatic resection}

A Korean retrospective study compared the results of sorafenib with hepatic resection in HCC patients with PVTT within the segmental branches and/or right and left portal vein [16]. In the total analysis of all cases enrolled between January 2000 and December 2011, the survival rate was higher in the hepatic resection group than in the sorafenib group (1-year rate: $63.6 \%$ vs. $32.3 \%$; 2 -year rate: $31.3 \%$ vs. $5.6 \%$ ). Similarly, in the subgroup analysis of the cases who were enrolled in the era of sorafenib (since January 2008), the survival time remained longer in the hepatic resection group than in the sorafenib group (median: 24.6 months vs. 4.1 months). This finding suggested that hepatic resection should be superior to sorafenib in the improvement of survival in HCC patients with PVTT. However, their generalisation might be limited due to the absence of detailed information regarding baseline characteristics and retrospective nature.

\section{Sorafenib alone versus sorafenib combined with other interventions}

\section{Sorafenib alone versus sorafenib +} cryotherapy

Preliminary studies explored the efficacy and safety of cryoablation for the treatment of unresectable HCC [30-33]. Recently, a Chinese RCT has evaluated the clinical benefit of cryotherapy as an adjunctive treatment modality in HCC patients with PVTT receiving sorafenib [15]. Besides the response rate, the combination of sorafenib with cryotherapy provided a significantly longer overall survival time (median: 12.5 months vs. 8.6 months), a longer time to progression (median: 9.5 months vs. 5.3 months), a higher clinical efficacy rate $(23 \%$ vs. $7.6 \%)$, and a higher disease control rate (65.4\% vs. 44.2\%). Multivariate Cox regression analysis also identified combined use of sorafenib and cryotherapy as the independent predictor of survival and time to progression. Thus, cryotherapy might be considered as an additional treatment option for HCC with PVTT, if sorafenib was employed.

\section{Sorafenib alone versus sorafenib + percutaneous radiofrequency ablation (RFA)}

An Italian RCT also explored whether or not the addition of RFA could improve the outcomes of sorafenib in HCC patients with PVTT and Child-Pugh class A [19]. The investigators reported a significantly higher survival rate in the combination group than in the sorafenib alone group (1-year rate: $60 \%$ vs. $37 \%$; 2 -year rate: $35 \%$ vs. $0 \%$ ). On the basis of this finding, RFA should be an auxiliary choice of therapy in patients with HCC with PVTT receiving sorafenib.

\section{Conclusions}

Although sorafenib is regarded as the standard treatment option for advanced HCC, its clinical efficacy in HCC patients with PVTT has been frequently challenged by other interventions, such as HAIC, TACE, radioembolisation, radiotherapy, and hepatic resection. However, the relevant evidence is of relatively low quality. Thus, further RCTs with head-to-head comparisons of sorafenib with other interventions should be conducted. In addition, the evidence from RCTs suggested that cryoablation or radiofrequency ablation provide an additional survival benefit to HCC patients with PVTT receiving sorafenib.

\section{Acknowledgments}

Xingshun Qi and Xiaozhong Guo are joint senior authors.

\section{Conflict of interest}

The authors declare no conflict of interest.

\section{References}

1. Ferlay J, Shin HR, Bray F, et al. Estimates of worldwide burden of cancer in 2008: GLOBOCAN 2008. Int J Cancer 2010; 127: 2893-917.

2. Tandon P, Garcia-Tsao G. Prognostic indicators in hepatocellular carcinoma: a systematic review of 72 studies. Liver Int 2009; 29: 502-10

3. EASL-EORTC clinical practice guidelines: management of hepatocellular carcinoma. J Hepatol 2012; 56: 908-43.

4. Llovet JM, Ricci S, Mazzaferro V, et al. Sorafenib in advanced hepatocellular carcinoma. N Engl J Med 2008; 359: 378-90.

5. Cheng AL, Kang YK, Chen Z, et al. Efficacy and safety of sorafenib in patients in the Asia-Pacific region with advanced hepatocellular carcinoma: a phase III randomised, double-blind, placebo-controlled trial. Lancet Oncol 2009; 10: 25-34.

6. Novi M, Lauritano EC, Piscaglia AC, et al. Portal vein tumor thrombosis revascularization during sorafenib treatment for hepatocellular carcinoma. Am J Gastroenterol 2009; 104: 1852-4.

7. Irtan S, Chopin-Laly X, Ronot M, et al. Complete regression of locally advanced hepatocellular carcinoma induced by sorafenib allowing curative resection. Liver Int 2011; 31: 740-3. 
8. Moroni M, Zanlorenzi L. Complete regression following sorafenib in unresectable, locally advanced hepatocellular carcinoma. Future Oncol 2013; 9: 1231-7.

9. Kermiche-Rahali S, Di Fiore A, Drieux F, et al Complete pathological regression of hepatocellular carcinoma with portal vein thrombosis treated with sorafenib. World I Surg Oncol 2013; 11: 171.

10. Cho JY, Paik YH, Lim HY, et al. Clinical parameters predictive of outcomes in sorafenib-treated patients with advanced hepatocellular carcinoma. Liver Int 2013; 33: 950-7.

11. Zhou L, Li J, Ai DL, et al. Enhanced therapeutic efficacy of combined use of sorafenib and transcatheter arterial chemoembolization for treatment of advanced hepatocellular carcinoma. J Clin Oncol 2014; 32 (suppl): e1512.

12. Marinelli S, Granito A, Terzi E, et al. Clinical predictors of response to sorafenib in patients with hepatocellular carcinoma. J Clin Oncol 2013; 31 (Suppl): e15149.

13. Cho JY, Paik YH, Lim HY, et al. Analysis of clinical parameters as predictor of outcome in sorafenib-treated patients with advanced hepatocellular carcinoma. Hepatology 2012; 56: 462A.

14. Nakazawa T, Hidaka H, Shibuya A, et al. Overall survival in response to sorafenib versus radiotherapy in unresectable hepatocellular carcinoma with major portal vein tumor thrombosis: propensity score analysis. BMC Gastroenterology 2014; 14: 84.

15. Yang Y, Lu Y, Wang C, et al. Cryotherapy is associated with improved clinical outcomes of sorafenib for the treatment of advanced hepatocellular carcinoma. Exp Therap Med 2012; 3: 171-80.

16. Lee JM, Jang BK, Chung WJ, et al. The survival outcomes of hepatic resection compared with transcatheter arterial chemoembolization for hepatocellular carcinoma with portal vein tumor thrombus. Hepatol Int 2014; 8: S22-3.

17. Kasai K, Sawara K, Suzuki K. Combination therapy of intra-arterial 5 -fluorouracil and systemic pegylated interferon alpha-2b for advanced hepatocellular carcinoma. J Clin Oncol 2013; 31 (Suppl 4): A216.

18. Song DS, Song MJ, Bae SH, et al. A comparative study between sorafenib and hepatic arterial infusion chemotherapy for advanced hepatocellular carcinoma with portal vein tumor thrombosis. J Gastroenterol 2015; 50: 445-54.

19. Giorgio A, Farella N, Di Sarno A, et al. Western trial comparing percutaneous radiofrequency of both hepatocellular carcinoma and the portal venous tumor thrombus plus sorafenib with sorafenib alone. J Hepatol 2011; 54: S542.

20. Sinclair P. A prospective randomized clinical trial on 90yttruim transarterial radioembolization (therasphere(registered trademark)) vs standard of care (sorafenib) for the treatment of advanced hepatocellular carcinoma with portal vein thrombosis. J Vasc Interv Radiol 2014; 25: 817.e20.

21. Lauro RM, Reggiani P, Caccamo L, et al. Laparoscopic thermal-ablation of $\mathrm{HCC}$ with microwave: preliminary experience and results. J Vasc Interv Radiol 2012; 23: 853.e27-8.

22. Llovet JM, Bruix J. Systematic review of randomized trials for unresectable hepatocellular carcinoma: chemoembolization improves survival. Hepatology 2003; 37: 429-42.

23. Bruix J, Sherman M. Management of hepatocellular carcinoma. Hepatology 2005; 42: 1208-36.
24. Chung GE, Lee JH, Kim HY, et al. Transarterial chemoembolization can be safely performed in patients with hepatocellular carcinoma invading the main portal vein and may improve the overall survival. Radiology 2011; 258: 627-34.

25. Sangro B, Inarrairaegui M, Bilbao Jl. Radioembolization for hepatocellular carcinoma. J Hepatol 2012; 56: 464-73.

26. Mazzaferro V, Sposito C, Bhoori S, et al. Yttrium-90 radioembolization for intermediate-advanced hepatocellular carcinoma: a phase 2 study. Hepatology 2013; 57: 1826-37.

27. Salem R, Mazzaferro V, Sangro B. Yttrium 90 radioembolization for the treatment of hepatocellular carcinoma: biological lessons, current challenges, and clinical perspectives. Hepatology 2013; 58: 2188-97.

28. LaPointe Rudow D, Brown Jr RS, Emond JC, et al. One-year morbidity after donor right hepatectomy. Liver Transplantation 2004; 10: 1428-31.

29. Lauro RM, Maggi U, Paone G, et al. Contrast enhanced ultrasound (CEUS): a new low-cost imaging procedure for hepatic artery and portal vein early complications after liver transplantation. Liver Transplantation 2009; 15: S223.

30. Yang Y, Wang C, Lu Y, et al. Outcomes of ultrasound-guided percutaneous argon-helium cryoablation of hepatocellular carcinoma. J Hepatobiliary Pancreat Sci 2012; 19: 674-84.

31. Wu B, Xiao YY, Zhang X, et al. Magnetic resonance imagingguided percutaneous cryoablation of hepatocellular carcinoma in special regions. Hepatobiliary Pancreat Dis Int 2010; 9: 384-92.

32. Shimizu T, Sakuhara Y, Abo D, et al. Outcome of MR-guided percutaneous cryoablation for hepatocellular carcinoma. J Hepatobiliary Pancreat Surg 2009; 16: 816-23.

33. Orlacchio A, Bazzocchi G, Pastorelli D, et al. Percutaneous cryoablation of small hepatocellular carcinoma with US guidance and CT monitoring: initial experience. Cardiovasc Intervent Radiol 2008; 31: 587-94.

Received: 3.02. 2015

Accepted: 10.04 .2015 